\title{
In situ Nanobeam Electron Diffraction of Bulk Metallic Glasses
}

Thomas C. Pekin ${ }^{1,2,3}$, Colin Ophus ${ }^{2}$, Christoph Gammer ${ }^{4}$, Burak Ozdol ${ }^{2}$, R.O. Ritchie ${ }^{1,3}$ and Andrew M. Minor ${ }^{1,2,3}$

1. Department of Materials Science \& Engineering, University of California, Berkeley, CA 94720, USA.

2. National Center for Electron Microscopy, Molecular Foundry, Lawrence Berkeley National Laboratory, Berkeley, CA 94720, USA.

3. Materials Science Division, Lawrence Berkeley National Laboratory, Berkeley, CA 94720, USA.

4. Erich Schmid Institute of Materials Science, Jahnstraße 12, Leoben, Austria, 8700.

Nanobeam electron diffraction (NBED) is a relatively new, versatile and powerful technique that has been used recently in the study of crystalline materials to measure strain, polarization and orientation [15]. These advances have been made possible through the development of high-speed direct electron detectors, which allow for the acquisition of hundreds of thousands of diffraction patterns that can be analyzed to extract a rich set of information. Previously, diffraction-based electron microscopy characterization techniques such as fluctuation electron microscopy (FEM) have been widely-utilized to analyze the structure of bulk metallic glasses [6-8]. In typical FEM studies hundreds of diffraction patterns are aggregated in order to make a measure of global medium range order. However, as this is a global rather than a local measurement, traditional electron microscopy techniques have not yielded information relevant to features on the nanometer scale. Here, we show through the application of fast detectors and new analysis techniques an extension to FEM in which NBED is used to map variance as well as local order spatially with nanometer resolution. Our results show that it is possible to map the spatial distribution of medium range order in an amorphous material in addition to the traditional global measure of amount of medium range order.

In addition to measuring local order, the acquisition of thousands of spatially resolved diffraction patterns allows for the determination of local strain in amorphous materials [9]. By calculating the first diffraction ring's elliptical deviation from a circle, accurate strain measurements can be made [10]. When used in combination with in situ deformation, this technique allows to measure the time-resolved evolution of the strain field and therefore to image local deformation events.

In this study, an annealed $\mathrm{Cu}_{46} \mathrm{Zr}_{46} \mathrm{Al}_{8}$ bulk metallic glass was scanned and NBED patterns were acquired. Using an analysis technique similar to [11], the order and degree of rotational symmetry in the diffraction patterns were measured. Representative diffraction patterns corresponding to high degrees of two-, three-, four-, and five-fold symmetry are shown in Figure 1. These diffraction patterns correspond to regions of local order in the sample, and are indicative of nanoscale structural inhomogeneities. The degree of rotational symmetry could then be used to map regions containing local order, with nanometer resolution. This research was only possibly due to high speed NBED and direct electron detectors with sensitivity for single electron counting, which enables new methods of materials characterization in metallic glasses.

References:

[1] VB Ozdol et al, Applied Physics Letters 106 (2015), p. 253107.

[2] D Carvalho et al, Scientific Reports 6 (2016), p. 28459. 
[3] E Rauch and M Véron, Materials Characterization 98 ( 2014), p. 1-9.

[4] TC Pekin et al, Scripta Materialia 146 (2018), p. 87-90.

[5] K Muller et al, Applied Physics Letters 101 (2012), p. 212110.

[6] PM Voyles, JM Gibson and MMJ Treacy, Journal of Electron Microscopy 49 (2000), p. 259-266.

[7] PM Voyles and D Muller, Ultramicroscopy 93 (2002), p. 147-159.

[8] PM Voyles and JR Abelson, Solar Energy Materials and Solar Cells 78 (2003), p. 85-113.

[9] C Gammer et al, Applied Physics Letters, Submitted.

[10] C Ebner et al, Ultramicroscopy 165 (2016), p. 51-58.

[11] A Liu et al. Physical Review Letters 110 (2013), p. 205505.

[12] The authors acknowledge support from the Director, Office of Science, Office of Basic Energy

Sciences, Materials Sciences and Engineering Division, of the U.S. Department of Energy under

Contract No. DE-AC02-05-CH11231 within the Mechanical Behavior of Materials program. Work at the Molecular Foundry was supported by the Office of Science, Office of Basic Energy Sciences, of the U.S. Department of Energy under Contract No. DE-AC02-05CH11231.
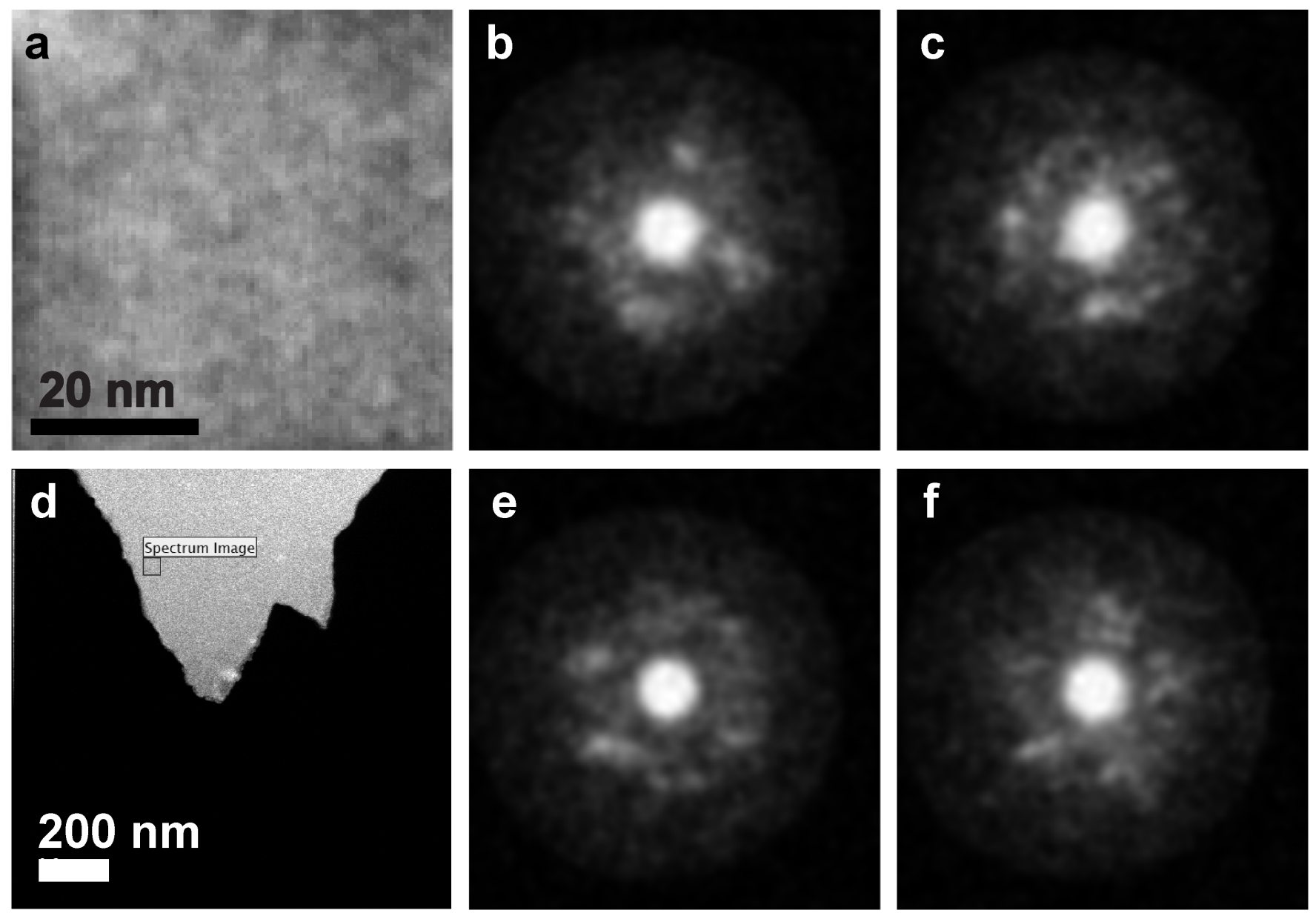

Figure 1. a) High angle annular dark field (HAADF) image of the region of interest scanned with NBED. b, c, e, f) Representative diffraction patterns exhibiting two-, three-, four-, and five-fold symmetry, respectively. d) Low magnification HAADF image showing lack of visible damage to the sample. 